\title{
QUALIDADE DE SABOR DE TOMATES DOS TIPOS SALADA E CEREJA E SUA RELAÇÃO COM CARACTERES MORFOAGRONÔMICOS DOS FRUTOS
}

\author{
Taste quality of salad and cherry tomatoes and their relationship with the morphoagronomic \\ characteristics of the fruits
}

\author{
Fábio Moreira Sobreira ${ }^{1}$, Fabricio Moreira Sobreira ${ }^{2}$, Gustavo Dias de Almeida ${ }^{2}$, \\ Ruimário Inácio Coelho ${ }^{3}$, Rosana Rodrigues ${ }^{4}$, Frederico de Pina Matta ${ }^{3}$
}

\begin{abstract}
RESUMO
Frutos no estádio vermelho maduro de trinta e três acessos de tomate do banco de germoplasma do CCA-UFES, sendo 15 do "tipo Cereja" e 18 do "tipo Salada", foram caracterizados com base nos seguintes descritores morfoagronômicos: peso médio do fruto; comprimento médio do fruto; diâmetro médio do fruto; diâmetro da cicatriz peduncular; espessura do mesocarpo do fruto; teor de sólidos solúveis e pH do fruto. Com base nas variáveis teor de sólidos solúveis (TSS) e pH do fruto, calculou-se a variável sabor, por meio da relação TSS $/ \mathrm{pH}^{2}$. Foram realizadas análises de variâncias e análises de correlações simples e parciais, determinando a distribuição dos acessos quanto às classes de sabor. Com base nas distribuições de frequências, formaram-se quatro classes para essa variável, sendo que, a maior parcela $(33 \%)$ dos acessos "tipo Salada" alocaram-se na classe mais baixa $\left(0,05-0,12{ }^{\circ} \mathrm{Brix}^{\mathrm{p}} \mathrm{pH}^{-2}\right)$, correspondendo a frutos de sabor menos desejável, enquanto a maior parcela $(30 \%)$ dos acessos "tipo Cereja", alocaram-se em classe imediatamente superior $\left(0,13-0,20^{\circ}{ }^{\circ}\right.$ rix. $\left.\mathrm{pH}^{-2}\right)$. Os dados demonstram a possibilidade de se obter ganhos genéticos para sabor concomitantemente ao peso, comprimento e espessura do mesocarpo dos frutos.
\end{abstract}

Termos para indexação: Lycopersicon esculentum, ganho genético, sólidos solúveis, germoplasma.

\section{ABSTRACT}

Fruits in the ripe red stage of thirty three tomato (Lycopersicon esculentum Mill.) accessions from CCA-UFES germplasm collection, being 15 "Cherry type" and 18 "Salad type", were characterized based on the following morphooagronomic descriptors: average fruit weight; average fruit length; average fruit diameter; peduncle scar diameter; fruit mesocarp thickness; soluble solids and fruit $\mathrm{pH}$. Based on the data from the soluble solids and fruit $\mathrm{pH}$, the flavor variable was calculated by the ratio TSS/pH $\mathrm{pH}^{2}$. Variance analysis and simple and partial correlations were calculated, and the accessions were clustered in flavor classes. Based on the statistical model of distribution of frequencies, four classes were formed for flavor variable. The largest portion (33\%) of the accessions of salad type were located in the lowest class $\left(0.05-0.12^{\circ} \mathrm{Brix}_{\mathrm{pH}} \mathrm{pH}^{-2}\right)$, corresponding to the accessions with not so desirable a flavor, while the largest portion (30\%) of the accessions of the "cherry" type were clustered in the next class above $(0.13-0.20$ ${ }^{\circ} \mathrm{Brix} . \mathrm{pH}^{-2}$ ). The possibility of concomitantly obtaining genetic gains for flavor, weight, length and mesocarp thickness of the fruits was demonstrated.

Index terms: Lycopersicon esculentum, genetic gain, soluble solids, germplasm.

(Recebido em 8 de outubro de 2008 e aprovado em 8 de maio de 2009)

\section{INTRODUÇÃO}

O fruto do tomateiro (Lycopersicon esculentum Mill.), atualmente é a principal hortaliça em termos de volume de produção e a segunda em termos de área plantada no Brasil (Food and Agricultural OrganizationFAO, 2007), sendo uma das principais fontes para o suprimento de diversos nutrientes importantes à dieta humana (Willcox et al., 2003).

A qualidade do tomate baseia-se, entre outros aspectos, nas suas características físico-químicas que tornam o produto preferido pelos consumidores, e sua qualidade nutricional, caracterizada pelo número de parâmetros responsáveis por propriedades ligadas à saúde humana (Anza et al., 2006).

O sabor do tomate é geralmente determinado pelo conteúdo de sólidos solúveis, acidez e presença de vários compostos voláteis ligados ao sabor dos frutos. Alguns desses compostos voláteis são produzidos por reações catalisadas por enzimas (Anthon \& Barrett, 2003). Segundo estudo de Jones \& Scott (1984), a maior contribuição para o sabor e consequente aceitabilidade, é dada pelos valores totais de açúcar e ácido encontrados nos frutos.

'Universidade Federal de Viçosa/UFV - 36570000 - Viçosa, MG - fabiomsobreira@yahoo.com.br

${ }^{2}$ Universidade Federal de Viçosa/UFV - Viçosa, MG

3Universidade Federal do Espírito Santo/UFES - Alegre, ES

${ }^{4}$ LMGV/UENF - Campos dos Goytacazes, RJ 
A análise sensorial pode eficientemente discriminar os acessos de um banco de germoplasma quanto ao sabor, porém, não é de uso prático por parte dos melhoristas, por requerer muitos julgadores treinados, necessários para várias semanas de avaliação (Colombani et al., 2001). O desenvolvimento de métodos para avaliar a qualidade dos frutos de tomate, com base em atributos químicos e físicos, vem a ser a alternativa viável e prática na classificação de acessos quanto ao sabor. Vários estudos foram realizados nesse sentido, porém, ainda não foi possível definir nenhum parâmetro ou relação ideal para classificar os mais diversos genótipos de tomate. Kader et al. (1978) estabeleceram como um bom indicador de sabor os teores de açúcar, acidez e a relação açúcar/acidez. Outro pesquisador sugere o uso da relação sólidos solúveis/ácidos tituláveis na discriminação do sabor dos frutos entre variedades (Stevens, 1972), enquanto outros sustentam a idéia de que o sabor do tomate melhora concomitantemente com a acidez e açúcar (Jones \& Scott, 1983).

A possibilidade de mensuração do sabor dos frutos por meio dos teores de sólidos solúveis e $\mathrm{pH}$ permitiu à elaboração de uma fórmula simples, composta pela razão entre o teor de açúcares e o quadrado do valor de $\mathrm{pH}$. De acordo com Guerra \& Zanus (2003), essa relação é mais precisa que a relação açúcar/acidez, (Santana et al., 2008) podendo, ser empregada para o acompanhamento da maturação dos frutos, visando à determinação da melhor época de colheita.

Conduziu-se este trabalho, com o objetivo de avaliar o uso da razão teor de sólidos solúveis e o quadrado do $\mathrm{pH}\left(\mathrm{TSS} / \mathrm{pH}^{2}\right)$ na classificação de acessos de tomate quanto ao sabor e identificar acessos que apresentem potencial para utilização em programas de melhoramento genético que visem ao aumento de produção com melhoria de sabor.

\section{MATERIAL E MÉTODOS}

O presente trabalho foi conduzido entre os meses de abril e agosto de 2006, em casa de vegetação no Centro de Ciências Agrárias da Universidade Federal do Espírito Santo (CCA-UFES), localizada a $20^{\circ} 45^{\prime} 48^{\prime \prime}$ de latitude sul e 41 $31^{\circ}$ '57" de longitude oeste de Greenwich e a $210 \mathrm{~m}$ de altitude, no município de Alegre - ES.

Foram utilizados 33 acessos de tomate do banco de germoplasma do CCA-UFES, sendo 18 acessos do "tipo Salada" e 15 acessos do "tipo Cereja” (Lycopersicon var. cerasiforme). Seis desses acessos, considerados do "tipo Cereja" (CCAUFES 77, CCAUFES 78, CCAUFES 79, CCAUFES 80, CCAUFES 81 e CCAUFES 82) são tipos comerciais. As sementes foram semeadas em bandejas de isopor de 128 células, utilizando-se o substrato Plantmax ${ }^{\circledR}$.
Aos 30 dias, após a semeadura, as plântulas foram transplantadas para vasos de $12 \mathrm{dm}^{3}$, preenchidos na proporção de 2:1:1 de terra, areia e esterco bovino curtido, respectivamente.

O delineamento experimental empregado foi blocos ao acaso, com três repetições e uma planta por parcela, sendo avaliados dez frutos por planta. As plantas foram tutoradas com fitilho e conduzidas com uma haste/planta (Marin et al., 2001). Os tratos culturais recomendados para a cultura foram realizados de acordo com Filgueira (2005).

Frutos no estádio vermelho maduro (mais de $90 \%$ da superfície vermelha) (Brasil, 2002), foram colhidos e imediatamente caracterizados com base nos seguintes descritores morfoagronômicos, de acordo com os descritores padrões, propostos pelo International Plant Genetic Resources Institute (International Plant Genetic Resources Institute-IPGRI, 1996): peso médio dos frutos em gramas (PMF), comprimento médio dos frutos (CMF) e diâmetro médio dos frutos (DMF), em $\mathrm{cm}$; diâmetro da cicatriz peduncular (DCP) e espessura do mesocarpo (ESP) em mm - mensurados com base na maior distância, utilizando paquímetro; teor de sólidos solúveis (TSS) em ${ }^{\circ}$ Brix - determinado por meio de leitura em refratômetro digital Atago modelo PR-101, com compensação de temperatura automática, e $\mathrm{pH}$ do fruto medido em $\mathrm{pHmetro}$ digital com eletrodo de membrana de vidro. Com base nos dados referentes ao teor de sólidos solúveis e $\mathrm{pH}$ dos frutos, calculou-se a "variável sabor" (SAB) pela fórmula $\mathrm{SAB}=\mathrm{TSS} / \mathrm{pH}^{2}$, sendo as análises realizadas no Laboratório de Biotecnologia do CCA-UFES.

Os dados obtidos foram submetidos à análise de variância univariada e, na existência de variabilidade genética, ao teste de Tukey, a 5\% de probabilidade. Para as características que apresentaram dificuldade de interpretação pelo referido teste, utilizou-se o teste de agrupamento proposto por Scott \& Knott (1974) a 1\% de probabilidade. Também foram realizadas análises de correlações simples (Pearson) e parciais, sendo todas as análises obtidas com base no programa computacional GENES (Cruz, 2001).

\section{RESULTADOS E DISCUSSÃO}

Os dados demonstraram diferenças significativas entre os acessos ao nível de $1 \%$ de probabilidade pelo Teste F, para todas as características avaliadas (Tabela 1). Destaca-se ainda o resultado das análises de variância isoladas, dos 18 acessos "tipo Salada" e dos 15 acessos "tipo Cereja". Esses resultados indicam presença de variabilidade genética entre os acessos para todas as variáveis analisadas. 
Tabela 1 - Resumos das análises de variância conjunta para os 33 acessos, e isolada para os 18 acessos "tipo Salada" e para os 15 acessos do "tipo Cereja", considerando as variáveis: peso médio do fruto (PMF; em g), comprimento médio do fruto (CMF; em cm), diâmetro médio do fruto (DMF; em cm), diâmetro da cicatriz peduncular (DCP; em mm), espessura do mesocarpo do fruto (ESP; em mm), teor de sólidos solúveis (TSS; em ${ }^{\circ} \mathrm{Brix}$ ), $\mathrm{pH}$ e "sabor" do fruto (SAB; em ${ }^{\circ} \mathrm{Brix}_{\mathrm{pH}}{ }^{-2}$ ), Alegre-ES, 2006.

\begin{tabular}{|c|c|c|c|c|c|c|c|c|}
\hline \multicolumn{9}{|c|}{ Anova para os 33 acessos $^{11}$} \\
\hline & PMF & CMF & DMF & $\mathrm{DCP}$ & ESP & TSS & $\mathrm{pH}$ & $\mathrm{SAB}$ \\
\hline Q.M. & $4841,98 * *$ & $3,73 * *$ & $5,75 * *$ & $34,75 * *$ & $7,14 * *$ & $3,44 * *$ & $0,06 * *$ & $0,01 * *$ \\
\hline Média & 34,93 & 3,63 & 3,65 & 5,68 & 4,33 & 2,76 & 4,35 & 0,150 \\
\hline C.V.(\%) & 58,56 & 19,34 & 15,87 & 21,16 & 13,07 & 23,73 & 3,37 & 27,520 \\
\hline $\mathrm{h}^{2}(\%)$ & 91,35 & 86,74 & 94,15 & 95,83 & 95,52 & 87,54 & 67,38 & 84,06 \\
\hline \multicolumn{9}{|c|}{ Anova para os 18 acessos tipo Salada } \\
\hline & PMF & CMF & DMF & DCP & ESP & TSS & $\mathrm{pH}$ & SAB \\
\hline Q.M. & $6935,32 * *$ & $3,71 * *$ & $5,45 * *$ & $39,89 * *$ & $5,39 * *$ & $1,20 * *$ & $0,05 * *$ & $0,004 * *$ \\
\hline Média & 51,59 & 4,09 & 4,43 & 7,40 & 5,14 & 2,30 & 4,40 & 0,120 \\
\hline C.V.(\%) & 54,01 & 19,47 & 17,05 & 20,86 & 12,80 & 26,91 & 3,07 & 28,230 \\
\hline$h^{2}(\%)$ & 88,81 & 82,96 & 89,54 & 94,02 & 91,97 & 68,09 & 61,07 & 72,4 \\
\hline \multicolumn{9}{|c|}{ Anova para os 15 acessos tipo Cereja } \\
\hline & PMF & CMF & DMF & DCP & ESP & TSS & $\mathrm{pH}$ & SAB \\
\hline Q.M. & $293,28 * *$ & $2,34 * *$ & $1,48 * *$ & $6,00 * *$ & $4,22 * *$ & $4,62 * *$ & $0,07 * *$ & $0,012 * *$ \\
\hline Média & 14,96 & 3,10 & 2,73 & 3,63 & 3,36 & 3,31 & 4,30 & 0,180 \\
\hline C.V.(\%) & 20,52 & 19,37 & 8,78 & 17,42 & 12,78 & 21,30 & 3,78 & 26,490 \\
\hline $\mathrm{h}^{2}(\%)$ & 96,79 & 84,55 & 96,12 & 93,34 & 95,64 & 89,22 & 64,61 & 81,05 \\
\hline
\end{tabular}

/1** significativo pelo teste $\mathrm{F}$ a $1 \%$ de probabilidade; Q.M. = Quadrados médios das análises de variâncias para os acessos avaliados; Média obtida para a respectiva variável; C.V. $(\%)=$ coeficiente de variação; $h^{2}(\%)=$ herdabilidade.

As elevadas estimativas de herdabilidade obtidas para "sabor" demonstram que a seleção direta dessa variável poderá promover o melhoramento genético do sabor dos frutos, pois este parâmetro corresponde à proporção da variabilidade total que é de natureza genética, ou o quociente entre a variância genética e a variância total (Wright, 1976; Falconer, 1987), sendo de relevada importância, em razão do seu valor preditivo, expressando a confiança do valor fenotípico como um guia para o valor genético, ou o grau de correspondência entre o valor fenotípico e o valor genético (Falconer, 1987).

Na tabela 2, demonstra-se a existência de correlações fenotípicas negativas entre a variável sabor e as variáveis PMF, CMF e ESP. No entanto, quando se considera análise de correlações parciais, que avaliam a associação entre duas variáveis, livre da influência das demais, observa-se a existência de valores positivos e significativos, indicando que a seleção para $\mathrm{SAB}$ proporcionará ganhos indiretos nas variáveis PMF, CMF e ESP, e vice-versa. A variável DMF apresenta correlação fenotípica positiva e significativa com a variável sabor. Contudo, a seleção visando a $\mathrm{SAB}$ pode provocar efeito indireto negativo no DMF, pois o mesmo apresenta correlação parcial negativa e significativa com SAB. Essas informações são relevantes para programas de melhoramento do tomateiro, pois permitem o desenvolvimento de frutos mais saborosos associados ao maior peso, comprimento e espessura de mesocarpo.

As correlações simples (fenotípicas) e parciais entre a variável $\mathrm{SAB}$ e as demais variáveis foram todas estatisticamente significativas (Tabela 2).

As correlações simples entre PMF X SAB $(-0,46)$, CMF X SAB $(-0,5)$ e ESP X SAB $(-0,63)$ indicam que frutos com maior espessura do mesocarpo, maior peso e maior comprimento, apresentaram menores valores para a variável sabor. Entretanto, com base nas correlações parciais, verificam-se possibilidades de se obter ganhos tanto para PMF, CMF, ESP e SAB desde que sejam selecionados dentre os frutos que obtiverem maiores PMF, CMF e ESP, os frutos com maiores valores de SAB. Contudo, para as variáveis DMF X SAB, a correlação simples foi de 0,42 e a parcial de -0,23. Esses resultados confirmam o que se observa nos mercados consumidores, 
onde há grande interesse pelos frutos de tomates conhecidos como "italianos", os quais, de acordo com Alvarenga (2004), possuem, geralmente, frutos mais saborosos, mais alongados, diâmetro reduzido e polpa espessa.

Os testes de médias e de agrupamento estão apresentados na Tabela 3. Ressaltam-se, em negrito, seus respectivos valores máximos e mínimos observados. Verifica-se que, para a variável SAB, formaram-se quatro grupos, sendo o grupo com o maior valor médio $(0,35$ ${ }^{\circ}$ Brix. $\mathrm{pH}^{-2}$ ) composto por apenas um acesso (CCAUFES 77). Este é um acesso "tipo Cereja" já encontrado no comércio, com frutos pequenos, estando alocado nos grupos com os menores valores para PMF, CMF e DMF $(8,42 \mathrm{~g} ; 2,29 \mathrm{~cm}$ e $2,40 \mathrm{~cm}$, respectivamente).

$\mathrm{O}$ segundo grupo de médias da variável $\mathrm{SAB}$, com valores variando de 0,20 a $0,26{ }^{\circ} \mathrm{Brix}_{\mathrm{pH}} \mathrm{pH}^{-2}$, foi composto por cinco acessos, dentre os quais, quatro são do "tipo Cereja" (CCAUFES 25, CCAUFES 64, CCAUFES 80, CCAUFES 81) e apenas um do "tipo Salada" (CCAUFES 63). É interessante destacar que o acesso CCAUFES 80, apesar de estar alocado no grupo com as maiores médias da variável TSS (5,38 a 6,61 ${ }^{\circ}$ Brix), enquadrou-se em um grupo distinto e inferior de sabor. Esse fato pode ter sido ocasionado pelos maiores valores de $\mathrm{pH}$, demonstrando que, com pequenas alterações nos valores de ${ }^{\circ}$ Brix ou de $\mathrm{pH}$ é possível discriminar os acessos em várias classes de sabor. $\mathrm{O}$ terceiro grupo de médias da variável SAB foi formado pelo maior número de acessos (15), sendo nove do "tipo Cereja" e seis do "tipo Salada".

O quarto grupo de médias da variável SAB foi composto pelos acessos de menor sabor, sendo onze do "tipo Salada" e apenas um do "tipo Cereja", indo ao encontro do esperado, pois frutos do tipo Salada tendem a ser menos saborosos. Nesse grupo merece comentário o acesso CCAUFES 83, conhecido como "tipo tomatão", pelo elevado valor de PMF (211,98 g) superior aos demais. Tamanha magnitude é explicada pela divergência genética da planta, cuja característica é produzir poucos frutos, mas com valores em peso, comprimento e diâmetro bem acima da média.

Com base na distribuição das frequências observadas, a "variável sabor", possibilitou a alocação dos acessos em quatro classes (Figura 1). Observa-se que $36 \%$ dos acessos avaliados foram alocados no intervalo compreendido entre 0,05 e $0,12^{\circ} \mathrm{Brix} . \mathrm{pH}^{-2}$, sendo $11(33 \%)$ do "tipo Salada" e apenas 1 (3\%) do "tipo Cereja".

No intervalo seguinte, compreendido entre 0,13 a 0,20 ${ }^{\circ} \mathrm{Brix} \mathrm{pH}^{-2}$, foram alocados $48 \%$ dos acessos, dos quais $10(30 \%)$ são do "tipo Cereja" e seis (18\%) do "tipo Salada". No intervalo compreendido entre 0,21 e 0,28 ${ }^{\circ}$ Brix.pH $\mathrm{pH}^{-2}$, foram alocados $12 \%$ dos acessos, sendo o "tipo Cereja" responsável por três acessos e o "tipo Salada" por apenas um. No intervalo correspondente à classe com maiores valores para a variável $\mathrm{SAB}$, foi encontrado apenas um acesso, sendo este do "tipo Cereja". Portanto, houve maior predominância dos acessos "tipo Salada" nas duas classes inferiores de sabor, ou seja, menor valor de ${ }^{\circ}$ Brix. $\mathrm{pH}^{-2}$. Por outro lado, os acessos "tipo Cereja", conforme era de se esperar, ocorreram com maior freqüência nas classes intermediárias e superiores, com os maiores valores de ${ }^{\circ} \mathrm{Brix} . \mathrm{pH}^{-2}$, demonstrando que os acessos deste tipo são mais saborosos, comparativamente aos acessos do "tipo Salada". Portanto, a fórmula apresentada para calcular a variável $\mathrm{SAB}$, presta-se satisfatoriamente na discriminação rápida e prática dos acessos de tomate quanto às classes de sabor.

Tabela 2 - Correlações entre as variáveis: peso médio do fruto (PMF; em g), comprimento médio do fruto (CMF; em cm), diâmetro médio do fruto (DMF; em $\mathrm{cm}$ ), diâmetro da cicatriz peduncular (DCP; em mm), espessura do mesocarpo do fruto

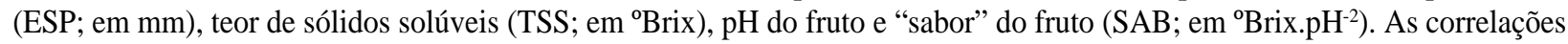
fenotípicas estão apresentadas acima da diagonal e, as correlações parciais, apresentadas abaixo da mesma.

\begin{tabular}{lllllll}
\hline & SAB & PMF & CMF & DMF & DCP & ESP \\
\hline SAB & & $-0,46^{*}$ & $-0,5^{*}$ & $0,42^{*}$ & $-0,46^{*}$ & $-0,63^{*}$ \\
PMF & $0,28^{*}$ & & $0,78^{*}$ & $0,92^{*}$ & $0,87^{*}$ & $0,58^{*}$ \\
CMF & $0,19^{*}$ & $0,30^{\mathrm{ns}}$ & & $0,84^{*}$ & $0,68^{*}$ & $0,75^{*}$ \\
DMF & $-0,23^{*}$ & $0,50^{\mathrm{ns}}$ & $0,89^{\mathrm{ns}}$ & & $0,89^{*}$ & $0,72^{*}$ \\
DCP & $0,05^{*}$ & $0,39^{\mathrm{ns}}$ & $-0,49^{\mathrm{ns}}$ & $0,34^{\mathrm{ns}}$ & & $0,62^{*}$ \\
ESP & $0,05^{*}$ & $-0,42^{\mathrm{ns}}$ & $0,46^{\mathrm{ns}}$ & $0,31^{\mathrm{ns}}$ & $-0,04^{\mathrm{ns}}$ & \\
\hline
\end{tabular}

*: Significativo e ${ }^{\text {ns: }}$ não significativo, ao nível de $5 \%$ de probabilidade. 
Tabela 3 - Médias apresentadas pelos 33 acessos em relação as variáveis: peso médio do fruto (PMF; em g), comprimento médio do fruto $(\mathrm{CMF}$; em cm), diâmetro médio do fruto (DMF; em $\mathrm{cm})$, diâmetro da cicatriz peduncular (DCP; em mm), espessura do mesocarpo do fruto (ESP; em mm), teor de sólidos solúveis (TSS; em ${ }^{\circ}$ Brix), pH do fruto (pH) e "sabor" do fruto (SAB; em ${ }^{\circ}$ Brix.pH $\left.{ }^{-2}\right)$, Alegre-ES, 2006.

\begin{tabular}{|c|c|c|c|c|c|c|c|c|}
\hline \multirow{2}{*}{$\begin{array}{c}\text { Acessos } \\
\text { CCAUFES }\end{array}$} & \multicolumn{8}{|c|}{ Variáveis } \\
\hline & $\mathrm{PMF}^{1 /}$ & $\mathrm{CMF}^{1 /}$ & $\mathrm{DMF}^{1 /}$ & $\mathrm{DCP}^{1 /}$ & $\mathrm{ESP}^{1 /}$ & $\mathrm{TSS}^{1 /}$ & $\mathrm{pH}^{2 /}$ & $\mathrm{SAB}^{1 /}$ \\
\hline $05(\mathrm{C})$ & $14,75 \mathrm{c}$ & $3,74 c$ & $2,54 \mathrm{~d}$ & $2,37 d$ & $2,95 \mathrm{~d}$ & $2,90 \mathrm{~b}$ & $4,19 \mathrm{ab}$ & $0,17 \mathrm{c}$ \\
\hline $09(\mathrm{~S})$ & $32,35 c$ & $3,30 \mathrm{c}$ & $4,13 \mathrm{c}$ & $8,77 \mathrm{~b}$ & $5,14 b$ & $3,02 b$ & $4,27 \mathrm{ab}$ & $0,16 \mathrm{c}$ \\
\hline $11(\mathrm{C})$ & $11,89 \mathrm{c}$ & $2,88 \mathrm{c}$ & $2,71 d$ & $4,26 c$ & $3,70 \mathrm{c}$ & $2,78 b$ & $4,61 \mathrm{a}$ & $0,13 \mathrm{c}$ \\
\hline $16(\mathrm{C})$ & $7,05 \mathrm{c}$ & $2,11 \mathrm{c}$ & $2,22 \mathrm{~d}$ & $4,51 \mathrm{c}$ & $2,24 \mathrm{e}$ & $2,70 \mathrm{~b}$ & $4,28 \mathrm{ab}$ & $0,15 \mathrm{c}$ \\
\hline $19(\mathrm{~S})$ & $19,57 \mathrm{c}$ & $3,18 \mathrm{c}$ & $3,14 \mathrm{c}$ & $4,20 \mathrm{c}$ & $4,29 \mathrm{c}$ & $2,45 b$ & 4,48ab & $0,12 d$ \\
\hline $21(\mathrm{~S})$ & $14,31 \mathrm{c}$ & $2,65 c$ & $2,90 \mathrm{~d}$ & $4,31 \mathrm{c}$ & $3,70 \mathrm{c}$ & $3,30 \mathrm{~b}$ & 4,44ab & $0,17 \mathrm{c}$ \\
\hline $25(\mathrm{C})$ & $13,44 \mathrm{c}$ & $2,83 \mathrm{c}$ & $2,79 d$ & $3,20 \mathrm{~d}$ & $3,13 d$ & $3,48 b$ & $4,12 b$ & $0,22 b$ \\
\hline $28(\mathrm{~S})$ & $18,27 \mathrm{c}$ & $3,41 \mathrm{c}$ & $3,09 \mathrm{c}$ & $2,81 \mathrm{~d}$ & $4,02 \mathrm{c}$ & $2,79 b$ & 4,18ab & $0,16 \mathrm{c}$ \\
\hline $29(\mathrm{~S})$ & $25,84 \mathrm{c}$ & $3,45 c$ & $3,42 \mathrm{c}$ & $6,51 \mathrm{c}$ & $4,05 \mathrm{c}$ & $2,53 b$ & 4,37ab & $0,13 \mathrm{c}$ \\
\hline $40(\mathrm{C})$ & $37,34 \mathrm{c}$ & $4,46 b$ & $4,18 \mathrm{c}$ & $4,50 \mathrm{c}$ & $5,58 b$ & $3,04 \mathrm{~b}$ & 4,41ab & $0,16 \mathrm{c}$ \\
\hline $46(\mathrm{~S})$ & $86,06 \mathrm{~b}$ & $4,84 \mathrm{~b}$ & $5,62 b$ & $14,92 \mathrm{a}$ & $6,11 b$ & $2,52 b$ & $4,51 \mathrm{ab}$ & $0,13 \mathrm{c}$ \\
\hline $48(S)$ & $21,33 \mathrm{c}$ & $3,03 \mathrm{c}$ & $3,49 \mathrm{c}$ & $5,74 \mathrm{c}$ & $4,26 \mathrm{c}$ & $1,41 \mathrm{c}$ & $4,22 \mathrm{ab}$ & $0,08 \mathrm{~d}$ \\
\hline $50(\mathrm{C})$ & $9,88 \mathrm{c}$ & $3,26 \mathrm{c}$ & $2,59 \mathrm{~d}$ & $2,61 \mathrm{~d}$ & $3,14 d$ & $2,81 b$ & 4,20ab & $0,16 \mathrm{c}$ \\
\hline $51(\mathrm{C})$ & $8,25 \mathrm{c}$ & $2,77 \mathrm{c}$ & $2,02 \mathrm{~d}$ & $2,74 \mathrm{~d}$ & $3,00 \mathrm{~d}$ & $2,90 \mathrm{~b}$ & 4,19ab & $0,16 \mathrm{c}$ \\
\hline $52(\mathrm{~S})$ & $21,25 c$ & $4,39 b$ & $5,01 \mathrm{~b}$ & $5,31 \mathrm{c}$ & $3,34 d$ & $2,62 b$ & $4,24 \mathrm{ab}$ & $0,15 c$ \\
\hline $55(\mathrm{~S})$ & $31,48 \mathrm{c}$ & $4,09 \mathrm{~b}$ & $3,87 \mathrm{c}$ & $4,24 \mathrm{c}$ & $5,23 b$ & $1,95 \mathrm{c}$ & 4,53ab & $0,09 d$ \\
\hline $63(\mathrm{~S})$ & $17,99 \mathrm{c}$ & $2,84 \mathrm{c}$ & $3,30 \mathrm{c}$ & $6,17 \mathrm{c}$ & $3,51 \mathrm{~d}$ & $3,68 b$ & $4,24 \mathrm{ab}$ & $0,21 b$ \\
\hline $64(C)$ & $26,42 \mathrm{c}$ & $4,77 \mathrm{~b}$ & $3,48 \mathrm{c}$ & $5,09 \mathrm{c}$ & $5,53 b$ & $3,49 \mathrm{~b}$ & $4,15 a b$ & $0,20 \mathrm{~b}$ \\
\hline $69(\mathrm{~S})$ & $28,78 \mathrm{c}$ & $3,30 \mathrm{c}$ & $3,87 \mathrm{c}$ & $5,05 \mathrm{c}$ & $5,49 \mathrm{~b}$ & $2,31 \mathrm{c}$ & $4,45 \mathrm{ab}$ & $0,12 \mathrm{~d}$ \\
\hline $71(\mathrm{C})$ & $33,54 \mathrm{c}$ & $3,72 \mathrm{c}$ & $3,98 \mathrm{c}$ & $6,99 \mathrm{c}$ & $4,73 b$ & $1,18 \mathrm{c}$ & $4,25 \mathrm{ab}$ & $0,06 \mathrm{~d}$ \\
\hline $72(\mathrm{~S})$ & $27,39 \mathrm{c}$ & $3,21 \mathrm{c}$ & $3,75 \mathrm{c}$ & $5,70 \mathrm{c}$ & $4,50 \mathrm{c}$ & $2,24 \mathrm{c}$ & $4,41 \mathrm{ab}$ & $0,11 \mathrm{~d}$ \\
\hline $77(\mathrm{C})$ & $8,42 \mathrm{c}$ & $2,29 \mathrm{c}$ & $2,40 \mathrm{~d}$ & $3,73 \mathrm{~d}$ & $2,31 \mathrm{e}$ & $6,61 \mathrm{a}$ & $4,37 \mathrm{ab}$ & $0,35 \mathrm{a}$ \\
\hline $78(\mathrm{C})$ & $8,60 \mathrm{c}$ & $2,82 \mathrm{c}$ & $2,25 d$ & $1,88 \mathrm{~d}$ & $3,11 \mathrm{~d}$ & $2,76 b$ & $4,48 \mathrm{ab}$ & $0,14 \mathrm{c}$ \\
\hline $79(\mathrm{C})$ & $13,39 \mathrm{c}$ & $3,86 \mathrm{c}$ & $2,32 d$ & $2,63 d$ & $3,63 \mathrm{c}$ & $3,13 b$ & 4,34ab & $0,17 \mathrm{c}$ \\
\hline $80(\mathrm{C})$ & $12,19 \mathrm{c}$ & $2,63 c$ & $2,77 d$ & $3,44 d$ & $3,03 d$ & $5,38 \mathrm{a}$ & $4,55 \mathrm{ab}$ & $0,26 \mathrm{~b}$ \\
\hline $81(\mathrm{C})$ & $2,69 c$ & $1,47 \mathrm{c}$ & $1,63 \mathrm{~d}$ & $1,71 \mathrm{~d}$ & $1,10 \mathrm{f}$ & $3,56 \mathrm{~b}$ & $4,09 \mathrm{~b}$ & $0,21 b$ \\
\hline $82(\mathrm{C})$ & $16,53 \mathrm{c}$ & $2,94 \mathrm{c}$ & $3,07 \mathrm{c}$ & $4,77 \mathrm{c}$ & $3,20 \mathrm{~d}$ & $3,01 \mathrm{~b}$ & $4,26 a b$ & $0,17 \mathrm{c}$ \\
\hline $83(\mathrm{~S})$ & $211,98 \mathrm{a}$ & $6,41 \mathrm{a}$ & $8,35 \mathrm{a}$ & $16,63 a$ & $4,67 b$ & $1,82 \mathrm{c}$ & 4,48ab & $0,09 d$ \\
\hline $84(\mathrm{~S})$ & $61,69 b$ & $6,17 \mathrm{a}$ & $4,53 b$ & $6,46 c$ & $5,93 b$ & $2,07 \mathrm{c}$ & $4,52 \mathrm{ab}$ & $0,10 \mathrm{~d}$ \\
\hline $85(\mathrm{~S})$ & $63,89 b$ & $4,71 b$ & $4,89 b$ & $9,83 b$ & $6,14 b$ & $1,50 \mathrm{c}$ & $4,40 \mathrm{ab}$ & $0,08 \mathrm{~d}$ \\
\hline $86(\mathrm{~S})$ & $73,08 b$ & $4,99 \mathrm{~b}$ & $5,18 b$ & $9,02 b$ & $7,09 a$ & $1,58 \mathrm{c}$ & $4,60 \mathrm{a}$ & $0,07 d$ \\
\hline $87(\mathrm{~S})$ & $96,04 b$ & $4,80 \mathrm{~b}$ & $5,84 b$ & $8,30 \mathrm{~b}$ & $7,65 a$ & $1,62 \mathrm{c}$ & $4,45 a b$ & $0,08 d$ \\
\hline $88(\mathrm{~S})$ & $77,33 b$ & $4,78 b$ & $5,35 b$ & $9,30 \mathrm{~b}$ & $7,42 \mathrm{a}$ & $1,99 \mathrm{c}$ & $4,50 \mathrm{ab}$ & $0,10 \mathrm{~d}$ \\
\hline
\end{tabular}

${ }^{1 /}$ Médias agrupadas pelo teste de Scott-Knott a $1 \%$ de probabilidade; ${ }^{2 /}$ Médias seguidas pela mesma letra, na mesma coluna, não diferem entre si, pelo teste Tukey a 5\% de probabilidade; (C) Acessos "tipo Cereja"; (S) Acessos "tipo Salada". 
Isolando os acessos "tipo Salada" em um segundo gráfico (Figura 2), observa-se que $44,44 \%$ destes foram alocados no intervalo compreendido entre 0,08 a 0,11 ${ }^{\circ} \mathrm{Brix}_{\mathrm{pH}} \mathrm{pH}^{-2}$.

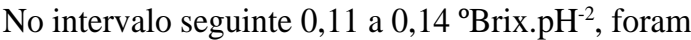
alocados $27,77 \%$ do total de acessos avaliados. No intervalo compreendido entre 0,14 a $0,17^{\circ} \mathrm{Brix} . \mathrm{pH}^{-2}$, foram alocados $22,22 \%$ dos acessos. No intervalo correspondente à classe com maiores valores para a variável SAB, foram encontrados, apenas, 5,55\% dos acessos. Verifica-se, portanto, que aproximadamente, $73 \%$ do total de acessos "tipo Salada" alocaram-se nas duas classes inferiores de sabor.
$\mathrm{Na}$ Figura 3, composta apenas por acessos "tipo Cereja", observa-se a distribuição dos acessos formando quatro classes.

Verifica-se que 13,33\% dos acessos "tipo Cereja" avaliados foram alocados no intervalo compreendido entre 0,06 a $0,13{ }^{\circ} \mathrm{Brix}_{\mathrm{pH}} \mathrm{pH}^{-2}$. No intervalo de 0,14 a $0,21^{\circ} \mathrm{Brix} \mathrm{pH}^{-2}$, estão inseridos 73,33\% do total de acessos "tipo Cereja" avaliados. No intervalo compreendido entre 0,22 a 0,29 ${ }^{\circ} \mathrm{Brix}_{\mathrm{pH}}{ }^{-2}$, agruparam-se 6,66\% dos acessos "tipo Cereja" e no intervalo correspondente a classe com maiores valores para a "variável" sabor, foram encontrados, apenas, 6,66\% dos acessos "tipo Cereja".

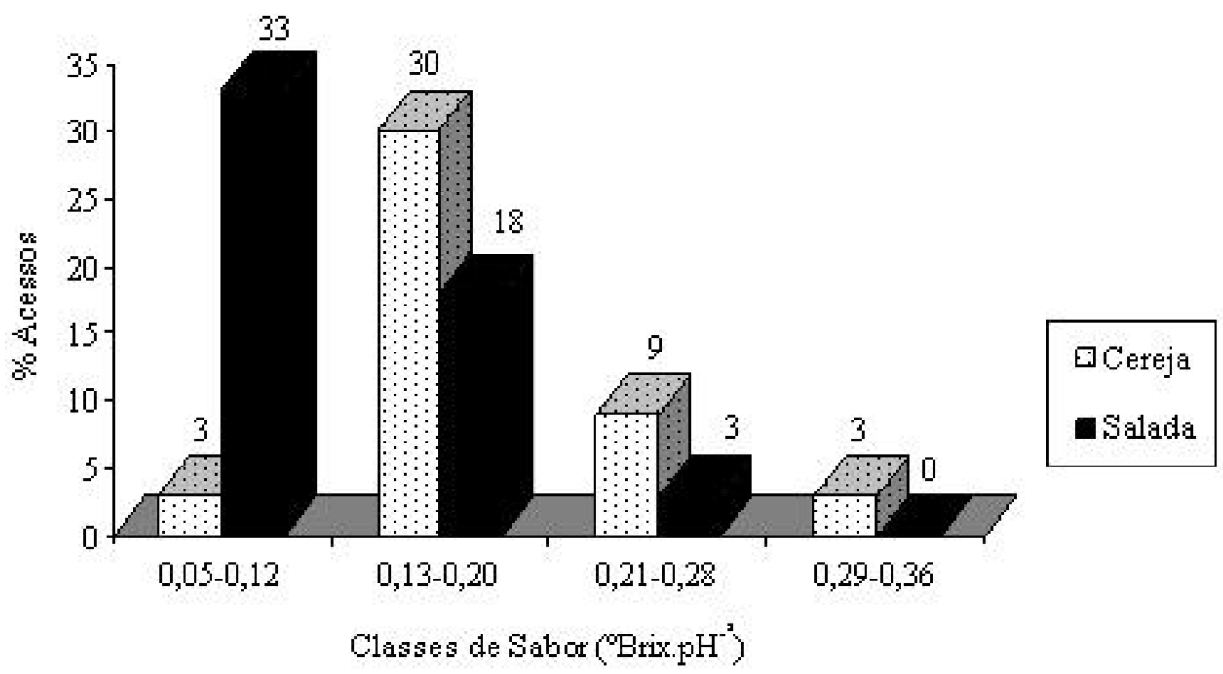

Figura 1- Frequência de 33 acessos de tomateiro dos tipos cereja e salada em diferentes classes para a variável sabor.

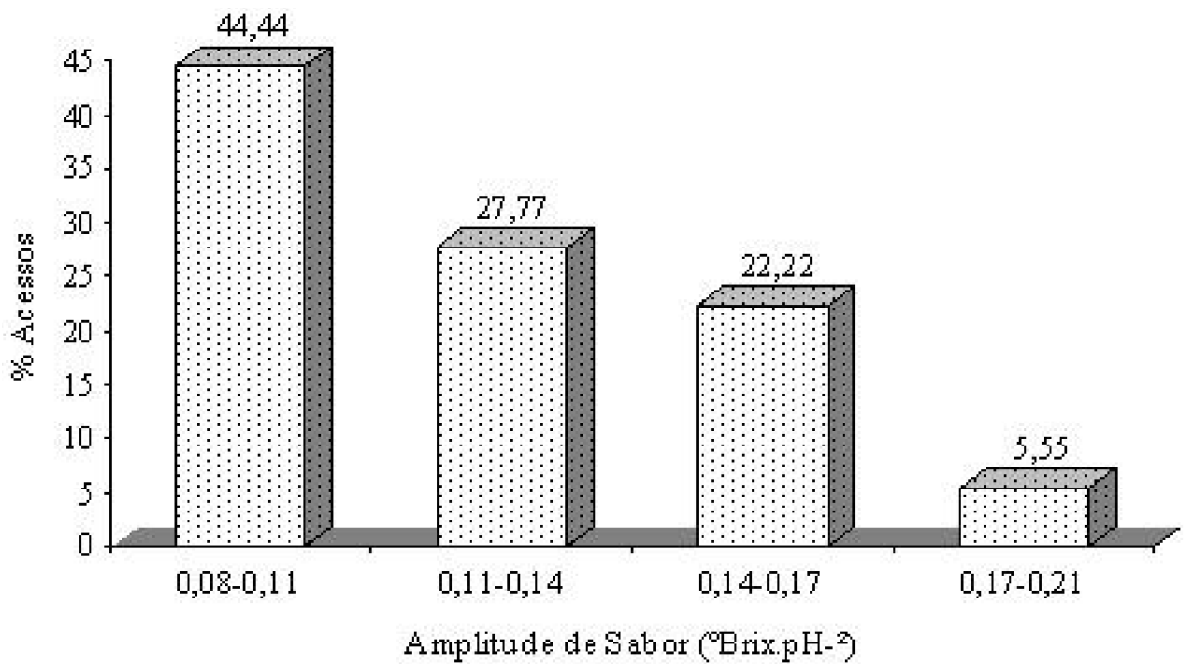

Figura 2- Frequência de 18 acessos de tomateiro do tipo Salada em diferentes classes para a variável sabor. 


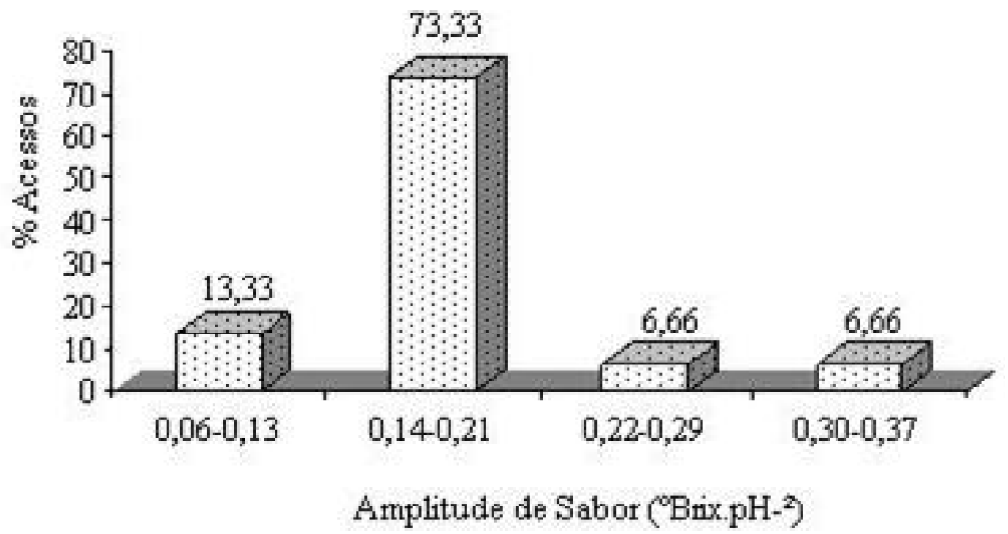

Figura 3 - Frequência de 15 acessos de tomateiro do tipo Cereja em diferentes classes para a variável sabor.

Portanto, este estudo demonstrou que o sabor dos frutos de tomate está fortemente ligado aos teores de açúcar e ácidos solúveis, conforme demonstrado anteriormente por Kader et al. (1977) e Stevens et al. (1977). O estudo do sabor, por meio das variáveis TSS e pH é de grande relevância, pois está sugerindo claramente existir uma relação ótima entre ácidos e açúcares em busca do que se chamaria de melhor sabor, ao ponto que um incremento ou redução em um desses fatores pode levar a efeitos negativos quanto à aceitabilidade pelos consumidores (Malundo et al., 1995).

Embora se saiba que compostos voláteis produzidos por reações catalisadas por enzimas também sejam responsáveis pelo sabor do fruto (Anthon \& Barrett, 2003) existem diferentes opiniões quanto à relativa importância destes (Barrett et al., 2007) e em razão dessa controvérsia, neste trabalho teve como objetivo avaliar a influência desses compostos na classificação dos acessos quanto à variável sabor.

A fórmula utilizada na variável $\mathrm{SAB}$, não visa a indicar com precisão os acessos mais saborosos aos consumidores, visto que a aceitabilidade é dependente de vários fatores, que incluem a população alvo identificada e amostrada (Malundo et al., 1995) e sim atuar como mais uma ferramenta para auxiliar o melhorista na seleção de acessos e/ou variáveis correlacionadas com as características de sabor.

\section{CONCLUSÕES}

O presente trabalho avaliou o uso da relação TSS/ $\mathrm{pH}^{2}$ na classificação dos acessos quanto ao sabor e demonstrou a possibilidade de uso desta, na discriminação dos acessos em distintas classes de sabor, conforme foi verificado pelo comportamento dos acessos "tipo Salada" em relação aos "tipo Cereja", sendo que estes localizaramse nos grupos superiores da variável SAB. Assim, com base nos 33 acessos do CCAUFES estudados e nos resultados obtidos pode-se concluir:

- A relação $\mathrm{TSS} / \mathrm{pH}^{2}$ é útil na discriminação dos acessos em distintas classes de sabor e na identificação das variáveis correlacionadas a este, podendo identificar de forma simplificada, quando comparada aos métodos tradicionalmente utilizados, os melhores acessos em um programa de melhoramento que tenha como objetivo a seleção para sabor dos frutos. Para esta finalidade, podem ser selecionados os acessos CCAUFES 77, CCAUFES 80, CCAUFES 25, CCAUFES 81, CCAUFES 63 e CCAUFES 64 que apresentaram os maiores valores para sabor.

- Ganhos para peso, comprimento, espessura e sabor dos frutos são possíveis desde que sejam selecionados dentre os frutos que apresentarem maiores peso, comprimento e espessura, os frutos com maiores valores de sabor. Ganhos indiretos podem ser obtidos, visto que a variável sabor apresenta correlação parcial positiva e significativa com as variáveis citadas. A seleção dos acessos CCAUFES 83, CCAUFES 84, CCAUFES 87 e CCAUFES 88 possibilita a obtenção de ganhos simultâneos para estas características.

\section{REFERÊNCIAS BIBLIOGRÁFICAS}

ALVARENGA, M.A.R. Tomate: produção em campo, em casa-de-vegetação e em hidroponia. Lavras: UFLA, 2004. $400 \mathrm{p}$.

ANTHON, A.; BARRETT, D.M. Thermal inactivation of lipoxygenase and hydroperoxytrienoiclyase in tomatoes. Food and Chemical Toxicology, Oxford, v.81, n.2, p.275$279,2003$. 
ANZA, M.; RIGA, P.; GARBISU, C. Effects of variety and growth season on the organoleptic and nutritional quality of hydroponically grown tomato. Journal of Food Quality, Ames, v.29, n.1, p.16-37, 2006.

BARRETT, D.M.; WEAKLEY, C.; DIAZ, J.V.; WATNIK, M. Qualitative and nutritional differences in processing tomatoes grown under commercial organic and conventional production systems JFS C: food chemistry and toxicology. Journal of Food Science, Chicago, v.72, n.9, p.441-451, 2007.

BRASIL. Ministério da Agricultura, Pecuária e Abastecimento. Portaria SARC n ${ }^{\circ} 085$, de 06 de março de 2002. Propõe o Regulamento técnico de identidade e qualidade para classificaçãodo tomate. Diário Oficial da República Federativa do Brasil, Brasília, mar. 2002.

COLOMBANI, V.S.; CAUSSE, M.; LANGLOIS, D.; PHILOUZE, J.; BURET M. Genetic analysis of organoleptic quality in fresh market tomato.1. Mapping QTLs for physical and chemical traits. Theoretical and Applied Genetics, Amsterdam, v.102, p.259-272, 2001.

CRUZ, C.D. Programa genes: versão windows: aplicativo computacional em genética e estatística. Viçosa, MG: UFV, 2001. 648p.

FALCONER, D.S. Introdução à genética quantitativa. Viçosa, MG: UFV, 1987. 279p.

FILGUEIRA, F.A.R. Novo manual de olericultura: agrotecnologia moderna na reprodução e na comercialização de hortaliças. Viçosa, MG: UFV, 2005. 402p.

FOOD AND AGRICULTURAL ORGANIZATION. Country information: Brazil: agriculture sector. 2007. Disponível em: 〈http://www. fao.org ago. 2008.

GUERRA, C.C.; ZANUS, M.C. Uvas viníferas para processamento em regiões de clima temperado. Brasília: Embrapa Uva e Vinho, 2003. CD-ROM.

INTERNATIONAL PLANT GENETIC RESOURCES INSTITUTE. Descriptors for tomato (Lycopersicon spp.). Roma, 1996. 47p.
JONES, R.A.; SCOTT, S.J. Genetic potential to improve tomato flavor in F[1] hybrids. Journal of American Society for Horticultural Science, Greensboro, v.109, n.3, p.318-321, 1984.

JONES, R.A.; SCOTT, S.J. Improvement of tomato flavor by genetically increasing sugar and acid contents. Euphytica, Wageningen, v.32, p.845-855, 1983.

KADER, A.A.; MORRIS, L.L.; STEVENS, M.A.; ALBRIGHT-HOLTON, M. Composition and flavor quality of fresh market tomatoes as influenced by some postharvest handling procedures. Journal of American Society for Horticultural Science, Greensboro, v.103, p.6-13, 1978.

KADER, A.A.; STEVENS, M.A.; ALBRIGHT-HOLTON, M.; MORRIS, L.L.; ALGAZI, M. Effect of fruit ripeness when picked on flavor and composition in fresh market tomatoes. Journal of American Society for

Horticultural Science, Greensboro, v.102, p.724-731, 1977.

MALUNDO, T.M.M.; SHEWFELT, A.R.L.; SCOTT, J.W. Flavor quality of fresh tomato (Lycopersicon esculentum Mill.) as affected by sugar and acid levels. Postharvest Biology and Technology, Wageningen, v.6, p.103-110, 1995.

MARIN, B.G.; SILVA, D.J.H.; GUIMARÃES, M.A.; BELFORT, G.; TEIXEIRA, M.B. Sistemas de condução de tomateiro visando produção na primavera e verão. Horticultura Brasileira, Brasília, v.19, p.227, 2001.

SANTANA, M. T. A.; SIQUEIRA, H. H. de; LACERDA, R. J.; LIMA, L. C. de O. Caracterisação fisico-quimica e enzimática de uva 'Patricia' cultivada na região de primavera do leste - MT. Ciência e Agrotecnologia, Lavras, v. 32 n. 1, p. 186-190, jan./fev., 2008.

SCOTT, A.J.; KNOTT, M. A cluster analysis method for grouping means in the analysis ofvariance. Biometrics, Arlington, v.30, p.507-512, 1974.

STEVENS, M.A. Relationships between components contributing to quality variation among tomato lines. Journal of American Society for Horticultural Science, Greensboro, v.97, p.70-73, 1972. 
STEVENS, M.A.; KADER, A.A.; ALBRIGHTHOLTON, M.; ALGAZI, M. Genotypic variation for flavor and composition in fresh market tomatoes. Journal of American Society for Horticultural Science, Greensboro, v.102, p.680689, 1977.
WILLCOX, J.K.; CATIGNANI, G.L.; LAZARUS, S.

Tomatoes and cardiovascular health. Critical Reviews in Food Science and Nutrition, v.43, p.1-18, 2003.

WRIGHT, J.W. Introduction to forest genetics. New York: Academic, 1976. 463p. 